TRANSACTIONS OF THE

AMERICAN MATHEMATICAL SOCIETY

Volume 191, 1974

\title{
POINTWISE DIFFERENTIABILITY AND ABSOLUTE CONTINUITY
}

\author{
BY
}

\author{
THOMAS BAGBY( ${ }^{1}$ ) AND WILLIAM P. ZIEMER( ${ }^{1}$ )
}

\begin{abstract}
This paper is concerned with the relationships between $L_{p}$ differentiability and Sobolev functions. It is shown that if $f$ is a Sobolev function with weak derivatives up to order $k$ in $L_{p}$, and $0 \leq l \leq k$, then $f$ has an $L_{p}$ derivative of order $l$ everywhere except for a set which is small in the sense of an appropriate capacity. It is also shown that if a function has an $L_{p}$ derivative everywhere except for a set small in capacity and if these derivatives are in $L_{p}$, then the function is a Sobolev function. A similar analysis is applied to determine general conditions under which the Gauss-Green theorem is valid.
\end{abstract}

1. Introduction. A fundamental result in real variable theory is that, if $f$ is an absolutely continuous function on a compact interval $l$, then $f^{\prime}$ exists almost everywhere and $\left|f^{\prime}\right|$ is integrable on $I$. The converse is false. However, if it is assumed that $f^{\prime}$ exists everywhere on $I$ and that $\left|f^{\prime}\right|$ is integrable, then $f$ is absolutely continuous. For a particularly simple proof of this, see [G]. Through a personal communication, we have learned that $C$. J. Neugebauer has improved this result by assuming merely that the approximate derivative of $/$ exists everywhere and that its absolute value is integrable.

In this paper we will be concemed with the higher dimensional analogues of these 1-dimensional concepts and we will establish results that contain those stated above. Thus, in our context, Sobolev functions in Euclidean $n$-space, $R^{n}$, will play the role of absolutely continuous functions in $R^{1}$ and the notion of derivative at a point will be taken in the sense introduced by Calderón and Zygmund [CZ]. In $\$ 3$ we discuss in a general setting the pointwise derivatives of Sobolev functions, including results with exceptional sets which are small in the sense of Hausdorff measure or capacity. These results extend theorems of Federer and Ziemer [FZ], Meyers [M2], and Serrin [SE]; the techniques we use are different, relying more on the original work of Calderón and Zygmund [CZ]. At the close of $\$ 3$ we prove that, if $u$ is a Sobolev function with weak derivatives up to order $k$, and $l$ is an

Received by the editors July 20, 1973.

AMS (MOS) subject classifications (1970). Primary 26A54; Secondary 46E35, 28 A75.

Key words and phrases. $L_{p}$ derivatives, Sobolev functions, capacity, normal currents, sets of finite perimeter, Gauss-Green theorem.

(1) This research was supported in part by National Science Foundation Grant GP33547. 
integer satisfying $0 \leq l \leq k$, then $u$ must agree with a function of class $C^{l}$ except for a set which is small in the sense of an appropriate capacity. In $\$ 4$ we discuss theorems of converse type, starting with ideas of Calderón [C2]. In particular, we prove that if a function has pointwise derivatives everywhere, except for a set which is small in the sense of measure or capacity, and these derivatives lie in appropriate $L_{p}$ classes, then the function must be a Sobolev function.

Since a characteristic property of absolutely continuous functions on the real line is that the fundamental theorem of calculus holds for them, another natural approach to the higher-dimensional problem is to take the Gauss-Green formula as the criterion for absolute continuity and also as a starting point for the definition of derivative at a point. We give results of this type in our final $\$ 5$, generalizing those obtained by S. Bochner [B] and V. Shapiro [SH].

2. Notation and preliminaries. Let $\mathcal{E}$ be a family of subsets of Euclidean $n$-space $R^{n}$. We say that two set functions $H_{1}: \mathcal{E} \rightarrow[0, \infty]$ and $H_{2}: \mathcal{E} \rightarrow[0, \infty]$ are equivalent if there exists a positive constant $C$ such that $C^{-1} H_{1}(E) \leq H_{2}(E)$ $\leq \mathrm{CH}_{1}(E)$ for $E \in \mathcal{E}$.

Now fix $t$ with $0 \leq t \leq n$. If $0<\delta \leq \infty$ we define the outer measure

$$
H_{(\delta)}^{t}(E)=\inf \left\{\frac{\Gamma(1 / 2)^{t}}{\Gamma(t / 2+1)} \sum_{j=1}^{\infty} 2^{-t}\left(\operatorname{diam} E_{j}\right)^{t}: E \subset \bigcup_{j=1}^{\infty} E_{j}, \operatorname{diam} E_{j} \leq \delta\right\}
$$

if $E \subset R^{n}$, and the $t$-dimensional Hausdorff outer measure $H^{t}(E)=\sup _{. \delta>0} H_{\delta}^{t}(E)$ if $E \subset R^{n}$. If in these definitions the sets $E_{j}$ are required to be cubes, we obtain the outer measure $Q_{(\delta)}^{t}$ and the $t$-dimensional cubical outer measure $Q^{t}$, respectively. It is clear that $H_{(\delta)}^{t}$ is equivalent to $Q_{(\delta)}^{t}$ and $H^{t}$ is equivalent to $Q^{t}$. If $0<\delta_{1}<\delta_{2}<\infty$ we have

$$
H_{(\infty)}^{t}(E) \leq H_{\left(\delta_{2}\right)}^{t} \leq H_{\left(\delta_{1}\right)}^{t}(E) \leq H^{t}(E), \quad E \subset R^{n},
$$

and, of course, the same inequalities hold with $Q$ in place of $H$; moreover, every cube with diameter $\leq \delta_{2}$ clearly can be decomposed into $\left(\left[\delta_{2} / \delta_{1}\right]+1\right)^{n}$ congruent cubes with diameter $\leq \delta_{1}$, and in this way every covering admissible in the definition of $Q_{\left(\delta_{2}\right)}^{t}(E)$ can be decomposed into a covering admissible in the definition of $Q_{\left(\delta_{1}\right)}^{t}(E)$; using Höldet's inequality, it follows that

$$
Q_{\left(\delta_{1}\right)}^{t}(E) \leq Q_{\left(\delta_{2}\right)}^{t}(E)\left(\left[\delta_{2} / \delta_{1}\right]+1\right)^{n-t}, \quad E \subset R^{n} .
$$

We conclude that if $\delta_{1}$ and $\delta_{2}$ are finite positive numbers, the outer measures $H_{\left(\delta_{1}\right)}^{t}$ and $H_{\left(\delta_{2}\right)}^{t}$ are equivalent; we therefore restrict our attention to $H_{(\infty)}^{t}$, $H_{(1)}^{t}$, and $H^{t}$ in the estimates obtained later in the paper. We mention one more equivalence: the trivial inequality $H_{(\infty)}^{t}(E)=H_{(\operatorname{diam} E)}^{t}(E)$ implies that, for a 
uniformly bounded family of sets $E, H_{(\infty)}^{t}$ and $H_{(1)}^{t}$ will be equivalent.

The outer measures $H_{(\infty)}^{t}$ and $H_{(1)}^{t}$ have the same null sets, as follows from (2.1) and the fact that, for any $\epsilon>0$, every set $E$ satisfying $H_{(\infty)}^{t}(E)<$ $\min \left\{\epsilon, 2^{t} \Gamma(1 / 2)^{-t} \Gamma(t / 2+1)\right\}$ must satisfy $H_{(1)}^{t}(E)<\epsilon$. The outer measures $H_{(1)}^{t}$ and $H^{t}$ have the same null sets, as follows from (2.1) and (2.2); however, smallness of $H_{(1)}^{t}$ does not imply smallness of $H^{t}$ for $0<t<n$, since $H^{t}(E)=\infty$ for arbitrarily small balls $E$.

The set functions $H_{(\delta)}^{t}$ and $H^{t}$ are generally defined only for $t \geq 0$, but in order to give unified statements of results we agree that for $t<0, H_{(\delta)}^{t}$ and $H^{t}$ assign infinite measure to each nonempty set.

If $\mu$ is a measure on a set $X, E \subset X$ is measurable, and $1 \leq p \leq \infty$, then $L_{p}(E, \mu)$ is the $L_{p}$-space defined by the restriction of $\mu$ to $E$. We let $\mathscr{L}_{n}$ denote Lebesgue measure on $R^{n}$, and we use the abbreviations $L_{p}(E)=L_{p}\left(E, \mathscr{L}_{n}^{n}\right)$, $L_{p}=L_{p}\left(R^{n}\right)$; we write $\|f\|_{p}=\|f\|_{L_{p}}$.

If $0 \leq k<\infty$ and $1 \leq p<\infty$, we consider the Banach space

$$
L_{p}^{k}=\left\{G_{k} * f: f \in L_{p}\left(R^{n}\right)\right\},
$$

where $G_{k} \in L_{1}\left(R^{n}\right)$ is defined by means of its Fourier transform: $\hat{G}_{k}(\xi)=$ $\left(1+4 \pi^{2}|\xi|^{2}\right)^{-k / 2}$. (See $[\mathrm{Cl}]$.) The norm in $L_{p}^{k}$ is given by $\left\|G_{k} * f\right\|_{L_{p}^{k}}=\|f\|_{p}$.

If $\Omega \subset R^{n}$ is open, we employ the usual notation for the Sobolev spaces $W_{p}^{k}(\Omega)=\left\{f: D^{a} f \in L_{p}(\Omega),|a| \leq k\right\}$ where $k$ is a nonnegative integer, $1 \leq p<\infty$, and $a=\left(a_{1}, a_{2}, \cdots, a_{n}\right)$ is a multi-index with $|\alpha|=\Sigma_{i=1}^{n} a_{i} \cdot W_{p}^{k}$ is a Banach space under the norm

$$
\|f\|_{W_{p}^{k}(\Omega)}=\sum_{|a|_{\leq k}}\left(\int\left|D^{a} f\right|^{p} d \S_{n}\right)^{1 / p} .
$$

(We note that the above derivatives are taken in the sense of distribution theory.) In case $\Omega=R^{n}$ and $p>1$ we have $W_{p}^{k}\left(R^{n}\right)=L_{p}^{k}$ and the two norms introduced above are equivalent [Cl Theorem 7].

If

$$
0 \leq k<\infty, \quad 1<p<\infty,
$$

we define for each $E \subset R^{n}$ the capacity $B_{k, p}(E)=\inf ,\|f\|_{p}^{p}$ where the infimum is taken over all nonnegative functions $f \in L_{p}$ such that $G_{k} * f \geq 1$ on $E$. It is proved in [Ml] that this capacity is an outer capacity; that is, $B_{k, p}$ is zero on the empty set, monotone, countable subadditive, and satisfies the regularity condition $B_{k, p}(E)=\inf \left\{B_{k, p}(G): G \supset E, G\right.$ open $\}$ where $E \subset R^{n}$ is arbitrary.

We denote by $B_{r}(x)$ the open ball of radius $r$ about the point $x$ in $R^{n}$. For each fixed $k$ and $p$ in the ranges (2.3) there exists a positive constant $C$ with the following property: 

$0<r \leq 1$.

(i) if $n-k p>0$, then $C^{-1} r^{n-k p} \leq B_{k, p}\left(B_{r}(x)\right) \leq C r^{n-k p}$ for $x \in R^{n}$,

(ii) if $n-k p=0$, then $C^{-1}(\log 1 / r)^{1-p} \leq B_{k, p}\left(B_{r}(x)\right) \leq C(\log 1 / r)^{1-p}$ for $x \in R^{n}, 0<r \leq 1 / 2$.

(iii) if $n-k p<0$, then $B_{k, p}(E) \geq C$ for every nonempty set $E \subset R^{n}$. Using these relations, we deduce at once that

$$
B_{k, p}(E) \leq C H_{(\infty)}^{n-k p}(E) \leq C H^{n-k p}(E) \text { if } E \subset R^{n},
$$

where $C$ is a positive constant depending only on $n, k$, and $p$.

We recall the spaces $T_{p}^{k}(x)$ and $t_{p}^{k}(x)$ which were introduced by Calderón and Zygmund [CZ]. If $1 \leq p \leq \infty$ and $k \geq-n / p, T_{p}^{k}(x)$ will denote those functions $f \in L_{p}$ for which there exists a polynomial $P_{x}$ of degree less than $k$ and a constant $M=M(x)$ such that for $0<r<\infty$

$$
\left(r^{-n} \int_{B_{r}(0)}\left|f(x+w)-P_{x}(w)\right|^{p} d \complement_{n}(w)\right)^{1 / p} \leq M r^{k} .
$$

When $p=\infty$, the left side is to be interpreted as ess sup $\left.\right|_{w}|<r| f(x+w)-P_{x}(w) \mid$. We note that in the limiting case $k=-n / p$ we have $f \in T_{p}^{k}(x)$ for every $f \in L_{p}$ and every $x \in R^{n}$, with $M=\|f\|_{p}$.

A function $f \in T_{p}^{k}(x)$ belongs to $t_{p}^{k}(x)$ if there is a polynomial $P_{x}$ of degree less than or equal to $k$ such that

$$
\left(r^{-n} \int_{B_{r}(0)}\left|f(x+w)-P_{x}(w)\right|^{p} d{ }^{\bigodot}(w)\right)^{1 / p}=o\left(r^{k}\right) \text { as } r \rightarrow 0 .
$$

In the event that $k$ is an integer, $f$ is said to possess a derivative of order $k$ in the $L_{p}$ sense.

The space $T_{p}^{k}\left(x_{0}\right)$ is a Banach space if for each $f \in T_{p}^{k}\left(x_{0}\right)$ we define the norm $\|f\|=T_{p}^{k}\left(x_{0}, f\right)$ to be the sum of $\|/\|_{p}$, the absolute values of the coefficients of the polynomial $P_{x}$, and the least admissible value of the constant $M$ in (2.5).

3. Pointwise derivatives of Sobolev functions. In this section we prove that functions in the space $L_{p}^{k}=\left\{G_{k} * f: f \in L_{p}\right\}$ must lie in the spaces $T_{\beta}^{l}\left(x_{0}\right)$ and $t_{\beta}^{l}\left(x_{0}\right)$, except for "small" sets of points $x_{0}$. We will study all values of $\beta$ for which the mapping

$$
L_{p}\left(R^{n}\right) \ni f \rightarrow G_{k} * f \in L_{\beta}\left(R^{n}\right)
$$

is continuous; and all values of $l$ up to the "order of differentiability" $k$ for which $T_{\beta}^{l}$ is defined, that is $-n / \beta \leq l \leq k$.

Theorem 3.1. Let $f \in L_{p}\left(R^{n}\right), 1<p<\infty$, and $k \geq 0$. Let $\beta$ satisfy 


$$
\begin{array}{ll}
p \leq \beta \leq n p /(n-k p) & \text { if } k p<n, \\
p \leq \beta<\infty & \text { if } k p=n, \\
p \leq \beta \leq \infty & \text { if } k p>n,
\end{array}
$$

and let $l$ satisfy $-n / \beta \leq l \leq k$.

(a) If $l \notin\{0,1,2, \ldots\}$, then for every $\epsilon>0$ there exists an open set $A$ with $H_{(1)}^{n-(k-l) p}(A)<\epsilon$, and a constant $C$, such that for all $x_{0} \in R^{n}-A$ we bave $T_{\beta}^{l}\left(x_{0}, G_{k} * f\right) \leq C$.

(b) If $l \notin\{0,1,2, \ldots\}$, then there exists a set $B$ with $H^{n-(k-l) p}(B)=0$ such that for all $x_{0} \in R^{n}-B$ we have $G_{k} * f \in t_{\beta}^{l}\left(x_{0}\right)$.

(c) If $l \in\{0,1,2, \cdots\}$, for every $\epsilon>0$ there exist an open set $D$ with $B_{k-l, p}(D)<\epsilon$, and a constant $C$, such that for all $x_{0} \in R^{n}-D$ we have $T_{\beta}^{l}\left(x_{0}, G_{k} * f\right) \leq C$.

(d) If $l \in\{0,1,2, \cdots\}$, there exists a set $E$ with $B_{k-l, p}(E)=0$ such that for all $x_{0} \in R^{n}-E$ we bave $G_{k} * f \in t_{\beta}^{l}\left(x_{0}\right)$.

Note. In the statement of parts (a) and (c) of the theorem the constant $C$ depends on $n, p, f, k, \beta, l$, and $\epsilon$, but is independent of $x_{0}$ and $r$. In case $l=k$ we are dealing with set functions $H_{(1)}^{n}, H^{n}$, and $B_{0, p^{\prime}}$ which are equivalent to Lebesgue outer measure; in this case the theorem is due to Calderón and Zygmund [CZ, pp. 204, 206]. At the other extreme we have $l \leq k-n / p$ (resp. $l<k-n / p$ ), when the exceptional sets in parts (a) and (b) (resp. parts (c) and (d)) are empty.

Part (d) of this theorem, with a different range of the parameters allowed, was obtained by Federer and $Z_{\text {iemer }}[\mathrm{FZ}]$ for $k=1$ and Meyers [M2] for general $k$, and by Serrin $[\mathrm{SE}]$ in a form involving Hausdorff measures. Part (b) of the theorem was obtained by Meyers [M2] in a form involving capacities. We will give a proof of the entire theorem which is quite different from these proofs and rests on the earlier work of Calderón and $Z_{y g m u n d}[\mathrm{CZ}]$.

Added in proof. After the present paper was submitted for publication, the authors learned that parts (b) and (d) of Theorem 3.1 are proved in the paper Maximal smoothing operators by A. P. Calderón, E. B. Fabes, and N. M. Riviere which will appear in Indiana Univ. Math. J.

Lemma 3.2. Let $\mu$ be a finite positive measure in $R^{n}$, and let $0<t \leq n$. Define

$$
\begin{aligned}
& M_{1}(x)=\sup _{0<r<\infty} \frac{1}{t^{t}} \mu\left(B_{r}(x)\right), \\
& M_{2}(x)=\sup _{0<r<1} \frac{1}{r^{t}} \mu\left(B_{r}(x)\right), \\
& M_{3}(x)=\limsup _{r \rightarrow 0} \frac{1}{r^{t}} \mu\left(B_{r}(x)\right) .
\end{aligned}
$$


Then there exists a constant $C$, depending only on $n$ and $t$, such that for every positive number $\sigma$ we have

(i) $H_{(\infty)}^{t}\left(\left\{x \in R^{n}: M_{1}(x)>\sigma\right\}\right) \leq C\|\mu\| / \sigma$,

(ii) $H_{(1)}^{t}\left(\left\{x \in R^{n}: M_{2}(x)>\sigma\right\}\right) \leq C\|\mu\| / \sigma$,

(iii) $H^{t}\left(\left\{x \in R^{n}: M_{3}(x)>\sigma\right\}\right) \leq C\|\mu\| / \sigma$.

Proof. We prove formula (i), since only minor changes in the proof are needed to obtain (ii) and (iii). Define $S=\left\{x \in R^{n}: M_{1}(x)>\sigma\right\}$. For each $x \in S$ we can find a ball $B_{r_{x}}(x)$ such that

$$
\mu\left(B_{r_{x}}(x)\right)>\sigma\left(r_{x}\right)^{t},
$$

and the family of all these balls is denoted by $\mathcal{F}$. We see $f$ rom (3.3) that the radii of balls in $\mathcal{F}$ are uniformly bounded by $\|\mu\|^{1 / t} \sigma^{-1 / t}$. Moreover, if $\left\{B_{r j}\left(x_{j}\right)\right\}_{j=1}^{\infty}$ is any disjoint sequence of balls in $\mathcal{F}$, then $\Sigma_{j=1}^{\infty}\left(r_{j}\right)^{t}<\sigma^{-1}\|\mu\|$, and hence the radii $r_{j} \rightarrow 0$. By a well-known covering argument (see [AS, $\left.\$ 10, \mathrm{p} .168\right]$ ) it is possible to find a disjoint (possibly finite) sequence of balls $B_{r_{j}}\left(x_{j}\right) \in \mathcal{F}$ such that $S \subset \bigcup_{x \in S} B_{r_{x}}(x) \subset \bigcup_{j} B_{S r_{j}}\left(x_{j}\right)$. Therefore

$$
H_{(\infty)}^{t}(S) \leq \frac{\Gamma(1 / 2)^{t}}{\Gamma(t / 2+1)} \sum_{j}\left(5 r_{j}\right)^{t} \leq \frac{\Gamma(1 / 2)^{t}}{\Gamma(t / 2+1} \varsigma^{t} \frac{\|\mu\|}{\sigma},
$$

as required.

From Lemma 3.2 and the estimates (2.4) we immediately deduce the foll owing:

Corollary 3.3. Let $\mu$ be a finite positive measure in $R^{n}$, and fix real numbers $k \geq 0$ and $p \geq 1$ with $k p<n$. Let

$$
M(x)=\sup _{0<r<\infty} \frac{1}{r^{n-k p}} \mu\left(B_{r}(x)\right) .
$$

For every positive constant $\sigma$, there exists an open set $\omega$ containing $\left\{x \in R^{n}\right.$ : $M(x)>\sigma\}$ such that $B_{k, p}(\omega) \leq C\|\mu\| / \sigma$.

The following well-know $n$ result may be deduced from Lemma 3.2 by the same arguments given in the book of Stein [S, Chapter 1] for the special case $k=0$.

Corollary 3.4. Let $f \in L_{p}$, and fix real numbers $k \geq 0$ and $p \geq 1$ with $k p<n$. Then there exists a set $E \subset R^{n}$ with $H^{n-k p}(E)=B_{k, p}(E)=0$ baving the following property:

(i) if $k>0$,

$$
\lim _{r \rightarrow 0} \frac{1}{r^{n-k p}} \int_{B_{r}(x)}|f|^{p} d £_{n}=0 \text { for all } x \in R^{n}-E .
$$

(ii) if $k=0$,

$$
\lim _{r \rightarrow 0} \frac{1}{r^{n}} \int_{B_{r}(x)}|f-f(x)|^{p} d \AA_{n}^{\varrho}=0 \text { for all } x \in R^{n}-E \text {. }
$$


Lemma 3.5. Let $f \in L_{p}\left(R^{n}\right), 1<p<\infty$, satisfy supp $f \subset B_{1}(0)$, and let $k \geq 0$ be arbitrary. Then for every $\epsilon>0$ there exists an open set $\omega$ with $B_{k, p}(\omega)<\epsilon$ and a constant $C$ such that $R_{k} *|f|\left(x_{0}\right)<C$ for all $x_{0} \in R^{n}-\omega$.

Proof. Since $G_{k} *|f| \in L_{p}^{k}$, it is well known that $G_{k} *|f|$ is bounded on $B_{2}(0)-\omega$, where $\omega$ is an open set satisfying $B_{k, p}(\omega)<\epsilon$ (see [Ml], Theorem 18]). It follows that $R_{k} *|f|$ is bounded on $B_{2}(0)-\omega$. But $R_{k} *|f|$ is clearly bounded on the complement of $B_{2}(0)$, since supp $f C B_{1}(0)$. This completes the proof.

Proof of Theorem 3.1. We begin with the proofs of parts (a) and (b). According to a theorem of Calderón and Zygmund [CZ, Theorem 4] convolution with the Bessel kernel $G_{k}$ gives a continuous linear transformation from $T_{p}^{w}\left(x_{0}\right)$ to $T_{\beta}^{w+k}\left(x_{0}\right)$,

$$
T_{p}^{w}\left(x_{0}\right) \ni f \rightarrow G_{k} * f \in T_{\beta}^{w+k}\left(x_{0}\right),
$$

for $1<p<\infty, k \geq 0$ and $\beta$ satisfying formulas (3.2), provided $w \geq-n / p$ and $w+k \notin\{0,1,2,3, \cdots\}$; the norm of the mapping (3.4) is independent of $x_{0}$. Moreover, the mapping (3.4) carries the subspace $t_{p}^{w}\left(x_{0}\right)$ into $t_{\beta}^{w+k}\left(x_{0}\right)$.

We now use the preceding result to prove parts $(a)$ and $(b)$ of the theorem. We begin with the case $l \geq k-n / p$. According to Lemma 3.2, for every $\epsilon>0$ we can find a constant $C \geq\|f\|_{p}^{p}$ and a set $A$ with $H_{(1)}^{n-(k-l) p}(A)<\epsilon$ such that $r^{-n+(k-l) p} \int_{B_{r}\left(x_{0}\right)}|f|^{p} d \bigodot_{n} \leq C$ for $x_{0} \in R^{n}-A$ and $0<r<1$, and thus for $x_{0} \in R^{n}-A$ and $0<r<\infty$ (this assertion is obvious if $n=p(k-l)$ ). Thus $T_{p}^{l-k}\left(x_{0}, f\right) \leq C^{1 / p}$ for these $x_{0}$; it follows that $T_{\beta}^{l}\left(x_{0}, G_{k} * f\right)$ is bounded for these $x_{0}$, if $\beta$ satisfies (3.2). Appealing to Corollary 3.4, we obtain for $l \neq k$ the estimate $r^{-n+(k-l) p} \int_{B_{r}\left(x_{0}\right)}|f|^{p} d \complement_{n}=o(1)$ as $r \rightarrow 0$, and for $l=k$ the

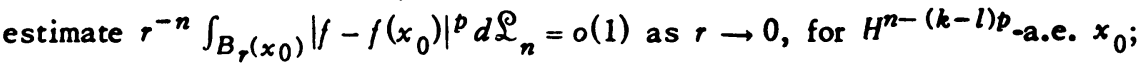
now $f \in t_{p}^{l-k}\left(x_{0}\right)$ for these $x_{0}$, so $G_{k} * f \in t_{\beta}^{l}\left(x_{0}\right)$ for these $x_{0}$ if $\beta$ satisfies (3.2).

Now suppose $l<k-n / p$. We distinguish two cases:

Case 1. If $k-n / p \notin\{0,1,2, \cdots\}$, we note that the hypothesis $f \in L_{p}$ implies that $T_{p}^{-n / p}\left(x_{0}, f\right)$ is bounded for all $x_{0} \in R^{n}$. It follows that $T_{\beta}^{k-n / p}\left(x_{0}, G_{k} * f\right)$ is bounded for all $x_{0}$, if $\beta$ satisfies (3.2). Since $-n / \beta \leq$ $l \leq k-n / p$ we conclude from [CZ, Lemma 2.1] that $T_{\beta}^{l}\left(x_{0}, G_{k} * f\right)$ is bound ed for all $x_{0}$ if $\beta$ satisfies (3.2). Similarly, $f \in t_{n}^{-n / p}\left(x_{0}\right)$ for all $x_{0} \in R^{n}$; using $\left[\mathrm{CZ}\right.$, Lemma 2.1] shows that $\bar{G}_{k} * f \in t_{\beta}^{k-n / p}\left(x_{0}\right) \subset t_{\beta}^{l}\left(x_{0}\right)$ if $\beta$ satisfies (3.2).

Case 2. If $k-n / p \in\{0,1,2, \cdots\}$, then $k p \geq n$, so for every $\beta$ satisfying (3.2) we can find an $\epsilon>0$ such that $k-n / p-\epsilon$ is a nonintegral real number in the interval $(l, k-n / p)$, and the mapping 


$$
T_{p}^{-n / p}\left(x_{0}\right) \ni g \rightarrow G_{k-\epsilon} * g \in T_{\beta}^{k-n / p-\epsilon}\left(x_{0}\right)
$$

is continuous and carries $t_{p}^{-n / p}\left(x_{0}\right)$ into $t_{\beta}^{k-n / p-\epsilon}\left(x_{0}\right)$. Applying this to function $g=G_{\epsilon} * f \in L_{p}$ we conclude that $T_{\beta}^{k-n / p-\epsilon}\left(x_{0}, G_{k} * f\right)$ is bounded for all $x_{0} \in R^{n}$. From [CZ, Lemma 2.1] we conclude that $T_{\beta}^{l}\left(x_{0}, G_{k} * f\right)$ is bounded for all $x_{0} \in R^{n}$. Similarly, $G_{\epsilon} * f \in t_{p}^{-n / p}\left(x_{0}\right)$ for all $x_{0} \in R^{n}$; again using [CZ, Lemma 2.1] shows that $G_{k} * f \epsilon t_{\beta}^{k-n / p-\epsilon}\left(x_{0}\right) \subset t_{\beta}^{l}\left(x_{0}\right)$.

Parts (c) and (d) of the theorem will be proved by means of the following result of Calderón and Zygmund [CZ, Theorem 11]. Let $1 \leq p<\infty, f \in L_{p}, k \geq 1, l \geq 1$, and let $\beta$ satisfy

$$
\begin{array}{ll}
p \leq \beta \leq n p /(n-p), & \text { if } p<n, \\
p \leq \beta<\infty, & \text { if } p=n, \\
p \leq \beta \leq \infty, & \text { if } p>n ;
\end{array}
$$

if $\partial\left(G_{k} * f\right) / \partial x^{j} \in T_{p}^{l-1}\left(x_{0}\right)$ for $j=1, \ldots, n$, then

$$
G_{k} * f \in T_{\beta}^{l}\left(x_{0}\right) \text { and } T_{\beta}^{l}\left(x_{0}, G_{k} * f\right) \leq C \sum_{j=1}^{n} T_{p}^{l-1}\left(x_{0}, \frac{\partial\left(G_{k} * f\right)}{\partial x^{j}}\right) \text {; }
$$

the constant $C$ is independent of $x_{0}$. Moreover, if $\partial\left(G_{k} * f\right) / \partial x^{j} \in t_{p}^{l-1}\left(x_{0}\right)$ for $j=1, \cdots, n$, then $G_{k} * f \in t_{\beta}^{l}\left(x_{0}\right)$ for $\beta$ satisfying (3.5). In view of these results, it suffices to prove (c) and (d) in the special case $l=0$.

If $k p>n$, and $f \in L_{p}$ it is well known that $G_{k} * f$ must be a continuous function; from the continuity of the mapping (3.1) we conclude that $T_{\beta}^{0}\left(x_{0}, G_{k} * f\right)$ is bounded for all $x_{0} \in R^{n}$, and $G_{k} * f \in t_{\beta}^{0}\left(x_{0}\right)$ for all $x_{0} \in R^{n}$, if $p \leq \beta \leq \infty$. Thus for the rest of the proof we assume $k p \leq n$ and $l=0$.

We can now prove part (c). If $f$ is supported by $\left\{x \in R^{n}:|x| \geq 1\right\}$ then by Hölder's inequality $G_{k} * f$ must be bounded on $R^{n}$, and hence $\left\|G_{k} * f\right\|_{L_{\beta}\left(B_{r}\left(x_{0}\right), P_{n} / r^{n}\right)}$ is bounded. Thus we may assume that $f$ is supported by $\left\{x \in R^{n}:|x| \leq 1\right\}$. Now by continuity of the mapping (3.1),

$$
\begin{aligned}
& \left\|G_{k} * f\right\|_{L_{\beta}\left(B_{r}\left(x_{0}\right), \mathcal{L}_{n} / r^{n}\right)} \leq C\left[\left\|G_{k} * f X_{B_{2 r}\left(x_{0}\right)}\right\|_{L_{\beta}\left(B_{r}\left(x_{0}\right), \mathcal{L}_{n} / r^{n}\right)}\right. \\
& +\| R_{k} * f\left(1-\chi_{B_{2 r}}\left(x_{0}\right) \|_{L_{\infty}\left(B_{r}\left(x_{0}\right)\right)}\right] \\
& \leq C\left[r^{k-n / p}\left\|f \chi_{B_{2 r}}\left(x_{0}\right)\right\|_{p}+2^{n-k}\left|R_{k} * f\left(x_{0}\right)\right|\right],
\end{aligned}
$$

where $X_{E}$ denotes the characteristic function of the set $E$; now part (c) follows from Corollary 3.3 and Lemma 3.5.

Part (d) may be proved by a computation like that just completed, but an even shorter proof results from the following remark on p. 198 of [CZ]: the implication $f \in t_{p}^{-k}\left(x_{0}\right) \Rightarrow G_{k} * f \in t_{\beta}^{0}\left(x_{0}\right)$ (which fails in general, as we noted at the 
beginning of our proof) will be valid if we know that $G_{k} *|f|\left(x_{0}\right)<\infty$. Since we know that the latter holds for $B_{k, p}$-q.e. $x_{0} \in R^{n}$, we see from part (b) that $\bar{G}_{k} * f \in t_{\beta}^{0}\left(x_{0}\right)$ for $B_{k, p}$-q.e. $x_{0} \in R^{n}$. This completes the proof of Theorem 3.1.

From Theorem 3.1 and an extension theorem of Calderón and Zygmund [CZ, Theorem 9] we may deduce a structure theorem for Sobolev functions $u \in L_{p}^{k}$ involving exceptional sets of small capacity. In fact, we know from Theorem 3.1 that, for every $\epsilon>0$, and every integer $l$ satisfying $0 \leq l \leq k$, we can find an open set $\omega$ with $B_{k-l, p}(\omega)<\epsilon$ such that $T_{p}^{l}\left(x_{0}, u\right) \leq C$ and $u \in t_{p}^{l}\left(x_{0}\right)$ for all $x_{0} \in R^{n}-\omega$. According to [CZ, Theorem 9] there exists a function $u_{\epsilon} \in C^{l}\left(R^{n}\right)$ such that $u(x)=u_{\epsilon}(x)$ for all $x \in R^{n}-\omega$. We summarize this result in the follow ing theorem.

Theorem 3.6. Let $u \in L_{p}^{k}, 1<p<\infty$, and let $l$ be an integer satisfying $0 \leq l \leq k$. For every $\epsilon>0$, there exist a function $u_{\epsilon} \in C^{l}\left(R^{n}\right)$ and an open set $\omega$ with $B_{k-l, p}(\omega)<\epsilon$, such that $u(x)=u_{\epsilon}(x)$ for all $x \in R^{n}-\omega$.

4. Theorems of converse type. This section is devoted to proving, in particular, that if a function $f$ is differentiable everywhere except on a sufficiently small set $K$ and if its derivatives are integrable, then $f$ is a Sobolev function. Thus we assume $f$ has a $k$ th order $L_{p}$ derivative almost everywhere, that the $L_{p}$ partial derivatives are in $L_{p}$, and that $f \in T_{p}^{k}(x)$ for all $x \in R^{n}-K$. We first prove that $f \in W_{p}^{k}\left(R^{n}\right)$, provided $K$ is a compact set whose projection on the coordinate hyperplanes is of $L_{n-1}$ measure zero; this proof relies on a result of A. P. Calderón [C]. We then give a completely different proof based on results from geometric measure theory $[F]$, which, in the case $k=1$, allows the hypothesis to be weakened.

We now let $\phi_{t}$ be a family of mollifiers defined for every $t>0$, by $\phi_{t}(x)=$ $t^{-n} \phi(x / t)$ where $\phi \in C^{\infty}$ whose support is contained in $B_{1}(0)$ and where $\int \phi d \mathscr{L}_{n}=1$.

Lemma 4.1. Let $f \in L_{p}\left(R^{n}\right), p \geq 1$, and suppose $f \in T_{p}^{k}(x)$ where $k$ is a positive integer. Then

$$
\liminf _{t \rightarrow 0^{+}} \phi_{t} * D^{a} f(y)>-\infty, \quad|x-y| \leq t,
$$

where $D^{\alpha} f$ denotes the distributional derivative of $f$ and $0 \leq|\alpha| \leq k$.

Proof. Since $f \in T_{p}^{k}(x)$ there is a polynomial $P_{x}$ of degree $k-1$ such that

$$
\int_{B(0, r)}\left|R_{x}(w)\right|^{p} d \mathcal{\complement}_{n}(w) \leq M(x) r^{n+p k}, \quad r>0,
$$


where $f(x+w)=P_{x}(w)+R_{x}(w)$. Let $F_{t}(y)=F(y, t)=\phi_{t} * f(y)$ and note that $D^{a} F_{t}(y)=\phi_{t} * D^{a} f(y)=D^{a} \phi_{t} * f(y)$. Therefore, $D^{a} F_{t}(y)=\int_{R^{n}} D^{a} \phi_{t}(w) f(y-w) d £_{n}(w)$. Thus,

$$
\begin{aligned}
& D^{a} F_{t}(x+b)=\int_{R^{n}} D^{\alpha} \phi_{t}(b-w) f(x+w) d \complement_{n}(w) \\
& =\int_{R^{n}} D^{a} \phi_{t}(b-w) P_{x}(w) d_{n}^{\varrho}(w)+\int_{R^{n}} D^{a} \phi_{t}(b-w) R_{x}(w) d \complement_{n}(w) .
\end{aligned}
$$

Clearly $f \in T_{p}^{l}(x)$ if $l \leq k$ and therefore, without loss of generality, we may assume $|\alpha|=k$. But then the first term in the last equality vanishes since $Q_{x}$ is of degree $k-1$. This leaves the last term which is the integral of a function which vanishes outside of the set $\{w:|b-w| \leq t\}$. Note we are considering only those $b$ for which $|b| \leq t$. Moreover, there is a constant $C$ such that $\left|D^{a} \phi_{t}(b-w)\right| \leq$ $\mathrm{Ct}^{-n-k}$. Consequently, reference to (4.1) yields

$$
\left|\int_{R^{n}} D^{a} \phi_{t}(b-w) R_{x}(w) d \mathcal{L}_{n}(w)\right| \leq C t^{-n-k} \int_{B_{2 t}(0)}\left|R_{x}(w)\right| d \mathcal{L}_{n}(w) \leq 2^{n+k} C M(x),
$$

and the lemma follows.

Now for a function $f \in t_{p}^{k}(x)$ we have relation (2.6) with a Taylor approximant $P_{x}(w)=\Sigma_{|a| \leq k}(1 / \alpha !) f_{\alpha}(x) w^{\alpha}$.

Corollary 4.2. If $f \in t_{p}^{k}(x)$, then for $|\alpha|=k$,

$$
\limsup _{t \rightarrow 0^{+}} \phi_{t} * D^{a} f(y)=f_{a}(x), \quad|x-y| \leq t .
$$

Proof. The proof is the same as in Lemma 4.1 except that

$$
D^{a} F_{t}(x+b)=f_{\alpha}(x)+\int_{R^{n}} D^{a} \phi_{t}(b-w) R_{x}(w) d \Sigma_{n}(w)
$$

because $D^{a} P_{x}(w)=f_{a}(x),|a|=k$. At this point the following result due to A. P. Calderón [C] becomes essential.

Theorem. Let $T$ be a distribution on $R^{n}$ and suppose $b$ is a locally integrable function such that, for $L_{n}$ almost every $x$,

$$
\limsup _{t \rightarrow 0^{+}} \phi_{t} * T(y) \geq b(x), \quad|x-y| \leq t,
$$

and for every $x$

$$
\lim _{t \rightarrow 0^{+}} \phi_{t} * T(y)>-\infty, \quad|x-y| \leq t
$$

Then, $T-b$ is a nonnegative Radon measure.

Now consider a function $f \in L_{p}$ and assume that $f \in T_{p}^{k}(x)$ for every $x \in \Omega$, where $\Omega$ is an open subset of $R^{n}$. Then it follows from the generalized 
Rademacher-Stepanov theorem [CZ, Theorem 5] that $f \in t_{p}^{l}(x)$, for $L_{n}$ almost every $x \in \Omega, 0 \leq l \leq k$. In view of Corollary 4.2, it follows that, for $L_{n}{ }^{-a . e .} x$,

$$
\lim _{t \rightarrow 0^{+}} \sup _{t} * D^{a} f(y)=f_{a}(x), \quad|x-y| \leq t, 0 \leq|a| \leq k .
$$

Consequently, Lemma 4.1 and Calderón's theorem imply that $D^{a} f-f_{a}$ is a nonnegative measure in the sense of distributions. However, this reasoning also applies to the function $-f$, and we conclude that $D^{\alpha}(-f)-\left(-f_{a}\right)$ is a nonnegative measure, or $D^{a} f-f_{a}$ is a nonpositive measure in $\Omega$. Thus

$$
D^{a} f=f_{a}, \quad 0 \leq|a| \leq k,
$$

in $\Omega$. If the functions $f_{a}$ are assumed to be in $L_{p}(\Omega)$, then $f \in W_{p}^{k}(\Omega)$.

Now let $K \subset R^{n}$ be a compact set and set $\Omega=R^{n}-K$. If $f \in T_{p}^{k}(x)$ for every $x \in \Omega$ and if the corresponding functions $f_{a}, 0 \leq|\alpha| \leq k$, are members of $L_{p}(\Omega)$, then

$$
f \in W_{p}^{k}\left(R^{n}\right)
$$

provided

$$
L_{n-1}\left[\Pi_{i}(K)\right]=0
$$

where the $\Pi_{i}: R^{n} \rightarrow R^{n-1}, i=1,2, \cdots, n$, are $n$ independent orthogonal projections of $R^{n}$ onto $R^{n-1}$. To see this, assume for the moment that the kernel $\Pi_{i}, i=1,2, \cdots, n$, are mutually orthogonal subspaces of $R^{n}$, thus determining a rectilinear coordinate system for $R^{n}$. Now, $f \in W_{p}^{k}(\Omega)$ and, in particular, $f \in W_{p}^{1}(\Omega)$. Therefore, $f$ contains a representative which is absolutely continuous on almost all line segments in $\Omega$ that are perpendicular to the coordinate $n-1$ planes. In view of the assumption (4.7) concerning $K, f$ in fact is absolutely continuous on almost all line segments in $R^{n}$, and thus is in $W_{p}^{1}\left(R^{n}\right)$ [MO, Chapter 3]. Similar reasoning applied to $D^{a_{1}} f,\left|\alpha_{1}\right|=1$, shows that $D^{\alpha_{1}} f \epsilon$ $W_{p}^{1}\left(R^{n}\right)$ and consequently, that $f \in W_{p}^{2}\left(R^{n}\right)$. Proceeding inductively, it follows that $f \in W_{p}^{k}\left(R^{n}\right)$. Recall that a function $f \in W_{p}^{k}\left(R^{n}\right)$ remains a Sobolev function if subjected to a linear, nonsingular change of coordinates. Thus, our assumption that the kernels $\Pi_{i}, i=1,2, \cdots, n$, are mutally orthogonal subspaces of $R^{n}$ is not restrictive and the proof of (4.6) is complete.

Thus, in summary we have

Theorem 4.3. Let $K \subset R^{n}$ be compact and suppose $f \in L_{p}\left(R^{n}\right)$ bas the property that $f \in T_{p}^{k}(x)$ for every $x \in R^{n}-K$. If $f_{a} \in L_{p}\left(R^{n}-K\right), 0 \leq|\alpha| \leq k$, and if. for $n$ independent orthogonal projections $\Pi_{i}, L_{n-1}\left[\Pi_{i}(K)\right]=0, i=1,2, \cdots, n$, then $f \in W_{p}^{k}\left(R^{n}\right)$. 
Now by employing entirely different techniques, we will prove Theorem 4.3, at least in the case $k=1$, under considerably weaker hypotheses. Indeed, we will weaken the hypothesis that $K$ is compact by requiring only that $K$ be a Borel set; we will replace the hypothesis that $f \in T_{p}^{1}(x)$ by requiring ap $\lim \sup _{b \rightarrow 0}|f(x+b)-f(x)| /|b|<\infty$, and we will drop the condition that $f \in L_{p}$. Our proof is based on results concerning functions whose partial derivatives are measures and we will use $[F]$ as a basic reference. In order to keep our exposition as brief as possible we will assume that the reader is well acquainted with the material in $\$ \$ 4.5 .6-4.5 .12$ in [F], and we will call upon results in these sections without giving complete details.

We say that a function $f$ is approximately differentiable at $x$ if we can write $f(x+y)=P_{x}(y)+R_{x}(y)$ where $P_{x}(w)$ is linear and if, for $\epsilon>0$, the set $\left\{y:\left|R_{x}(y) \geq \epsilon\right| y \mid\right\}$ has 0 as a point of dispersion.

We next show that $L_{p}$ differentiability implies approximate differentiability.

Lemma 4.4. If $f \in t_{p}^{1}(x), p \geq 1$, then $f$ is approximately differentiable at $x_{\text {. }}$

Proof. Suppose $\left(r^{-n} \int_{B_{r}(0)}\left|R_{x}(y)\right|^{p} d \mathscr{\complement}_{n}(y)\right)^{1 / p}=o(r)$ and let, for each $\epsilon>0$,

$$
E_{\epsilon}=\left\{y:\left|R_{x}(y)\right| \geq \epsilon|y|\right\} \text {. }
$$

Then, reference to $[\mathbf{N}$, Lemma 3], yields a constant $C$ such that

$$
\begin{aligned}
& O(r)=\left(r^{-n} \int_{B_{r}(0)}\left|R_{x}(y)\right|^{p} d \complement_{n}(y)\right)^{1 / p} \\
& \geq\left(r^{-n} \int_{E_{\epsilon} \cap B_{r}(0)}(\epsilon|y|)^{p} d £_{n}(y)\right)^{1 / p} \\
& \geq C\left(\left(r^{-n} \epsilon^{p} \mathcal{L}_{n}\left(E_{\epsilon} \cap B_{r}(0)\right)^{(n+p) / n}\right)^{1 / p}\right. \\
& =C \epsilon^{p}\left(\frac{\mathfrak{L}_{n}\left(E_{\epsilon} \cap B_{r}(0)\right)}{r^{n}}\right)^{1 / p} \mathscr{L}_{n}\left(E_{\epsilon} \cap B_{r}(0)\right)^{1 / n} .
\end{aligned}
$$

Thus, 0 is a point of dispersion for (4.8) and we have, for $i=1, \cdots, n$,

$$
a p \partial f / \partial x_{i}=f_{a_{i}}, \quad\left|a_{i}\right|=1
$$

Recall that, if $a p \lim \sup _{b \rightarrow 0}|/(x+b)| /|b|<\infty$ for every $x$ in some set $A$, then $f$ is approximately differentiable at a.e. point in $A,[F, 3.1 .8]$.

Theorem 4.5. Let $K \subset R^{n}$ be a Borel set and suppose $f: R^{n} \rightarrow R^{1}$ bas the property that ap lim $\sup _{b \rightarrow 0}|f(x+b)-f(x)| /|b|<\infty$ for eacb $x \in R^{n}-K$. If the approximate partial derivatives of $f$ are in $L_{p}^{10 c}, p \geq 1$, and if for $n$ independent 
orthogonal projections $\Pi_{i}, L_{n-1}\left[\Pi_{i}(K)\right]=0, i=1,2, \cdots, n$, then $f \in W_{p}^{1, \text { boc }}\left(R^{n}\right)$.

Proof. Without loss of generality, we may assume that $/$ has compact support.

For $x=\left(x_{1}, \cdots, x_{n}\right)$, define $f^{*}: R^{n} \rightarrow R^{n+1}$ by

$$
f^{*}(x)=\left(x_{1}, \cdots, x_{n}, f(x)\right)
$$

and define, as in $[F, 4.5 .9]$,

$$
\begin{aligned}
\lambda(x) & =a p \underset{y \rightarrow x}{\lim \inf } f(y), \\
\mu(x) & =a p \underset{y \rightarrow x}{\lim \sup } f(y), \\
C & =R^{n+1} \cap\left\{y: \mu\left(y_{1}, \ldots, y_{n}\right) \geq y_{n+1} \geq \lambda\left(y_{1}, \ldots, y_{n}\right)\right\} .
\end{aligned}
$$

Since ap lim sup $b \rightarrow 0$. $|(x+b)=f(x)| /|b|<\infty$ for $x \in R^{n}-K$ and the approximate derivatives of $f \stackrel{b \rightarrow 0}{\text { are }}$ in $L_{p}, p \geq 1$, it follows from [F, 3.1.8, 2.10.43, and 3.2.5] that there is a countable number of measurable set $S_{j}$ such that $f$ is Lipschitz on each $S_{j}, R^{n}-K=\bigcup_{j=1}^{\infty} S_{j}(2)$ and

$$
\infty>H^{n}\left[\bigcup_{j=1}^{\infty} f^{*}\left(S_{j}\right)\right] \text {. }
$$

Since $f$ is approximately continuous on $R^{n}-K$, note that

$$
C=\bigcup_{j=1}^{\infty} f^{*}\left(s_{j}\right) \cup B
$$

with $p(B)=K$ where $p: R^{n+1} \rightarrow R^{n}$ is the projection $p\left(y_{1}, \cdots, y_{n+1}\right)=\left(y_{1}, \cdots, y_{n}\right)$.

As in the proof of Theorem 4.3, it is sufficient to assume that the projections $\Pi_{i}: R^{n} \rightarrow R^{n-1}$ are of the form

$$
\Pi_{i}(x)=\left(x_{1}, \ldots, x_{i-1}, x_{i+1}, \ldots, x_{n}\right), \quad i=1, \ldots, n .
$$

Let $p_{i}: R^{n+1} \rightarrow R^{n}$ be $p_{i}(y)=\left(y_{1}, \cdots, y_{i-1}, y_{i+1}, \cdots, y_{n+1}\right), i=1, \cdots, n+1$. Since we are assuming $L_{n-1}\left[\Pi_{i}(K)\right]=0, i=1, \ldots, n$, it follows from (4.10), (4.11), and $[F, 3.2 .20]$ that

$$
\int_{R^{n}} N\left[p_{i} \mid C, z\right] d L_{n}(z)<\infty, \quad i=1, \ldots, n+1,
$$

where $N\left[p_{i} \mid C, z\right]$ denotes the cardinality of the set $p_{i}^{-1}(z) \cap C$. Again following $[F, 4.5 .9]$ we define

$$
G=R^{n+1} \cap\left\{y: \mu\left(y_{1}, \cdots, y_{n}\right) \geq y_{n+1}\right\}, \quad S=(-1)^{n} \partial\left(R^{n+1} L G\right) .
$$

(2) It can easily be shown this would still be valid if we retained the assumption that $f \in T_{p}^{1}(x)$ for every $x \in R^{n}-K$. 
The reasoning in the proof of $[F, 4.5 .9(5)]$ demonstrates that $C$ contains the essential boundary of $G$ and inspection of the proof of Theorem 4.5.11 in [F] shows (4.12) is sufficient to ensure that $S$ is a locally normal current. It is now easy to complete the proof when $f$ is bounded, for then $[F, 4.5,12]$ shows that $R^{n} L f \in N_{n}^{l o c}\left(R^{n}\right)$, i.e., $f$ locally is a function whose partial derivatives are measures. By assumption, $L_{n-1}\left[\Pi_{i}(K)\right]=0, i=1,2, \cdots, n$, and therefore $L_{n}\left[p_{i}(B)\right]=0, i=1,2, \cdots, n+1$. Thus it follows from the proof of $[F, 4.5 .9(29)]$ and $[F, 4.5 .9(27)]$ that $f$ is continuous and of bounded variation on almost every line parallel to the coordinate axes. Also, recall that we have shown that $f$ is Lipschitz on each $S_{j}$ where $R^{n}-K=\bigcup_{j=1}^{\infty} S_{j}$. Consequently, on every line which does not intersect $K, f$ carries sets of $L_{1}$ measure zero into sets of $L_{1}$ measure zero, thus establishing that $f$ is absolutely continuous on almost every line parallel to the coordinate axes. Since we are assuming the approximate partial derivatives $a p \partial f / \partial x_{i}$ are in $L_{p}$, it now follows that $f \in W_{p}^{1}\left(R^{n}\right)$, provided $f$ is bounded.

To remove the hypothesis that $f$ is bounded, we now let $f$ be any function satisfying the hypotheses of the theorem, and introduce the truncations

$$
f_{j}(x)= \begin{cases}j & \text { if } f(x)>j \\ f(x) & \text { if }-j \leq f(x) \leq j, \\ -j & \text { if } f(x)<-j\end{cases}
$$

Applying the above arguments we conclude that each $f_{j} \in W_{p}^{1}\left(R^{n}\right)$, and hence by Sobolev's inequality $\left\|f_{j}\right\|_{p} \leq C\left\|\nabla f_{j}\right\|_{p} \leq C\|a p \nabla f\|_{p}$. By Fatou's lemma,

$$
\|f\|_{p}^{p}=\int \lim _{j \rightarrow \infty}\left|f_{j}\right|^{p} d \mathscr{\complement}_{n} \leq \liminf _{j \rightarrow \infty} \int\left|f_{j}\right|^{p} d \complement_{n} \leq C^{p}\left\|_{a p} \nabla f\right\|_{p}^{p}
$$

Consequently, by Lebesgue's dominated convergence theorem,

$$
\int\left|f_{j}-f\right|^{p} d \complement \rightarrow 0 \text { and } \int\left|\nabla f_{j}-a p \nabla f\right|^{p} d \propto \rightarrow 0 .
$$

Thus, we conclude that $f \in W_{p}^{1}\left(R^{n}\right)$.

5. The Gauss-Green theorem. In this section we conclude the paper by developing another situation in which differentiability on a sufficiently large set implies absolute continuity. Our results generalize those of S. Bochner [B] and V. Shapiro [SH]: our methods are quite different. Here we consider a measurable vector field $v: R^{n} \rightarrow R^{n}$ such that at every point upper and lower divergences are defined, $\operatorname{div}^{*} v(x)$ and $\operatorname{div}_{*} v(x)$. Under the assumption that the upper and lower divergences are finite everywhere and that they are integrable, we show that $\operatorname{div}^{*} v(x)=\operatorname{div}_{*} v(x)$ for a.e. $x$ and that $\operatorname{div}^{*} v$ equals the distributional 
divergence of $v$. We also prove that when $\operatorname{div}^{*} v$ is employed, the Gauss-Green theorem is valid for almost every set whose boundary is given by the level set of a smooth function. If, in addition, $v$ is taken to be continuous, then the Gauss-Green theorem is shown to be valid for any open set whose boundary has finite $H^{n-1}$ measure, thus establishing the "absolute continuity" of $v$.

Let $\Omega \subset R^{n}$ be open and suppose $v: \Omega \rightarrow R^{n}$ is a vector field that is in $L_{p}(\Omega), 1 \leq p<\infty$. We will say that a closed $n$-dimensional interval $I$ is admissible for $v$ if the integral

$$
\int_{\partial I} v(x) \cdot \nu(x) d H^{n-1}(x)
$$

exists and is finite. Here $\nu(x)$ denotes the unit exterior normal to $I$ at $x$ and note that almost all intervals $l \subset \Omega$ are admissible for $v$. For every admissible $l \subset \Omega$, set $\mu(l)$ equal to the integral in (5.1) and define, for $x \in \Omega, \operatorname{div}^{*} v(x)=$ lim sup $\mu(I) / \aleph_{n}(I)$ where the lim sup is taken over a regular family of admissible intervals $I$ containing $x$ [SA, p. 106]. Define $\operatorname{div}_{*} v(x)$ as the corresponding lim inf and if $\operatorname{div}_{*} v(x)=\operatorname{div}^{*} v(x)$ is finite this common value will be called $\operatorname{div} v(x)$.

Lemma 5.1. Suppose $\infty>\operatorname{div}^{*} v(x) \geq \operatorname{div}_{*} v(x)>-\infty$ for every $x \in \Omega$ and assume that both $\operatorname{div}^{*} v$ and $\operatorname{div}_{*} v$ are integrable over $\Omega$. Then, for each admis. sible interval $I \subset \Omega, \int_{I} \operatorname{div}^{*} v d \varrho_{n} \geq \mu(I) \geq \int_{I} \operatorname{div}_{*} v d \varrho_{n}$.

Proof. Suppose from some admissible $I_{0} \subset \Omega$ and $\epsilon>0$ that

$$
\int_{I_{0}} \operatorname{div}^{*} v d \mathscr{C}_{n}<\mu\left(I_{0}\right)-\epsilon \cdot \mathscr{L}_{n}\left(I_{0}\right) .
$$

Let $f$ be a lower semicontinuous function such that $\operatorname{div}^{*} v(x) \leq f(x), x \in \Omega$, and

$$
\int_{\mathbb{Q}}\left(f-\operatorname{div}^{*} v\right) d \complement_{n}<\epsilon L_{n}\left(I_{0}\right) .
$$

For every admissible $I \subset \Omega$, let $\theta(I)=\int_{I} f d \mathscr{\varrho}_{n}-\mu(I)$, and observe that, in view of the lower semicontinuity of $f, \theta_{*}(x) \geq f(x)-\operatorname{div}^{*} v(x) \geq 0$ for every $x \in \Omega$. Thus, it follows easily that $\theta(l) \geq 0$ for every admissible $I \subset \Omega$ [SA, p. 190]; therefore from (5.2) and (5.3)

$$
\mu\left(I_{0}\right) \leq \int_{I_{0}} f d \mathscr{L}_{n} \leq \int_{I_{0}} \operatorname{div}^{*} v d \mathscr{\complement}_{n}+\mathcal{L}_{n}\left(I_{0}\right)<\mu\left(I_{0}\right),
$$

a contradiction. Thus, $\int_{l} \operatorname{div}^{*} v d \AA_{n} \geq \mu(l)$ for each admissible $l \subset \Omega$ and similar reasoning yields the remaining inequality of the lemma.

We will now proceed to prove that under the hypotheses of Lemma 5.1, div $v$ exists a.e. in $\Omega$ and that the divergence of $v$ in the sense of distributions is equal to $\operatorname{div} v$, that is, 


$$
\int v \cdot \nabla \phi d L_{n}=\int \operatorname{div} v \phi d \mathscr{L}_{n}
$$

for every $\phi \in C_{0}^{\infty}(\Omega)$.

To this end, denote by $A$ the family of all half-open intervals $J=\left\{x: a_{i} \leq\right.$ $\left.x<b_{i}, i=1,2, \cdots, n\right\}$ whose closures are admissible in $\Omega$. Let $\mathcal{F}$ denote the field of all finite unions of intervals $J \in A$, and note that $\mathcal{F}$ generates the Borel sets in $\Omega$. Now define a set function $\psi$ on $A$ by $\psi(J)=\mu(I)$ where $I$ is the closure of $J$. Then $\psi$ is finitely additive on $A$ and a theorem due to B. Fuglede is now applicable, [FU, Theorem III]:

In order that there exists a function $f \in L_{1}(\Omega)$ such that $\psi(J)=\int_{J} f d \varrho_{n}$ for every $J \in A$, the following two conditions are necessary and, when combined, sufficient:

(i) For every $\epsilon>0$ there is a $\delta>0$ sucb that $\sum_{i=1}^{k}\left|\psi\left(J_{i}\right)\right| \leq \epsilon$ for every finite number of intervals $J_{1}, \ldots, J_{k}$ from $A$ for whicb $\sum_{i=1}^{k} \mathscr{L}_{n}\left(J_{i}\right)<\delta$.

(ii) There is a constant $C$ sucb that $\sum_{i=1}^{k}\left|\psi\left(J_{i}\right)\right| \leq C$ for every finite system of disjoint intervals $J_{1}, \cdots, J_{k}$ from $A$.

If the hypotheses of Lemma 5.1 are in force, then clearly the lemma implies that conditions (i) and (ii) are satisfied and consequently

$$
\mu(I)=\int_{I} f d \AA_{n} \text { for every admissible } I \subset \Omega \text {. }
$$

Moreover, Lebesgue's theorem on differentiation shows $\operatorname{div} v(x)=f(x)$ for a.e. $x \in \Omega$. Thus, we have

Lemma 5.2. Under the bypotheses of Lemma 5.1, $\operatorname{div} v$ exists a.e. in $\Omega$ and $\int_{I} \operatorname{div} v d \mathfrak{L}_{n}=\int_{\partial I} v \cdot \nu d H^{n-1}$ for almost every closed interval $I \subset \Omega$.

Let $\Omega^{\prime}$ be an open set whose closure is contained in $\Omega$ and choose an arbitrary interval $l \subset \Omega^{\prime}$. As in $\$ 4$, we will employ the mollifier $\phi_{t^{\prime}}$, and it should be understood that only those $t>0$ for which $t$ is less than the distance from $\Omega^{\prime}$ to boundary $\Omega$ will be considered. We will define

$$
(\operatorname{div} v)_{t}=\operatorname{div} v * \phi_{t}
$$

and $v_{t}$ will denote the vector field whose coordinate functions are those of $v$ convolved with $\phi_{t}$.

With the aid of Lemma 5.2 and Fubini's theorem, we have

$$
\begin{aligned}
\int_{I}(\operatorname{div} v)_{t}(x) d \varrho_{n}(x) & =\int_{I} \int_{R^{n}} \operatorname{div} v(x-y) \phi_{t}(y) d £_{n}(y) d \varrho_{n}(x) \\
& =\int_{R^{n}} \int_{I_{y}} \operatorname{div} v(x) \phi_{t}(y) d £_{n}(x) d \varrho_{n}(y) \\
& =\int_{R^{n}} \int_{\partial I} v(x) \cdot \nu(x) \phi_{t}(y) d H^{n-1}(x) d \varrho_{n}(y)
\end{aligned}
$$


where $I_{Y}=I-Y$. On the other hand an application of the classical Gauss-Green theorem yields $\int_{I} \operatorname{div} v_{t}(x) d \mathscr{\complement}_{n}(x)=\int_{\partial I} v_{t}(x) \cdot \nu(x) d H^{n-1}$ and by Fubini's theorem, this is easily seen to be

$$
\int_{R^{n}} \int_{\partial I_{y}} \nu(x) \cdot \nu(x) \phi_{t}(y) d H^{n-1}(x) d £_{n}(y) .
$$

Therefore, for every $I \subset \Omega^{\prime}, \int_{I} \operatorname{div} v_{t} d \mathscr{S}_{n}=\int_{I}(\operatorname{div} v)_{t} d \mathscr{\complement}_{n}$ thus proving that

$$
\operatorname{div} v_{t}=(\operatorname{div} v)_{t} \text { on } \Omega^{\prime}
$$

Now let $\phi \in C_{0}^{\infty}(\Omega)$ and let $\Omega^{\prime}$ be an open set as above that contains the support of $\phi$. From (5.7) and Fubini's theorem we have

$$
\begin{aligned}
& \int \operatorname{div} v \phi d \varrho_{n}=\lim _{t \rightarrow 0^{+}} \int(\operatorname{div} v)_{t} \phi d \AA_{n}=\lim _{t \rightarrow 0^{+}} \int \operatorname{div} v_{t} \phi d \AA_{n} \\
& =\lim _{t \rightarrow 0^{+}} \int v_{t} \cdot \nabla \phi d £_{n}=\int v \cdot \nabla \phi d £_{n}
\end{aligned}
$$

which proves (5.4).

We now supplement (5.4) by showing that the Gauss-Green formula is valid for almost all level sets of the test function $\phi \in C_{0}^{\infty}(\Omega)$ :

$$
\int_{\phi^{-1}(y)} v \cdot \nu d H^{n-1}=\int_{\{\phi>y\}} \operatorname{div} v d \AA_{n} \text { for a.e. } y \in R^{1} \text {. }
$$

To prove this we will make use of the coarea formula [F, Theorem 3.2.12]:

$$
\int_{R^{n}} g(x)|\nabla \phi(x)| d \varrho_{n}(x)=\int_{R^{1}} \int_{\phi^{-1}(y)} g(x) d H^{n-1}(x) d £_{1}(y)
$$

if $g \in L_{1}\left(R^{n}\right)$.

First note that for a.e. $y \in R^{1}, \phi^{-1}(y)$ is a smooth manifold and is the boundary of $\{\phi>y\}$. Thus the classical Gauss-Green theorem is applicable to the smooth vector field $v_{t}$ :

$$
\int_{\{\phi>y\}} \operatorname{div} v d \AA_{n}=\lim _{t \rightarrow 0} \int_{\{\phi>y\}} \operatorname{div} v_{t} d \AA_{n}=\lim _{t \rightarrow 0} \int_{\phi^{-1}(y)} v_{t} \cdot \nu d H^{n-1} .
$$

We will now show for a subsequence $t_{k}$ that, for a.e. $y \in R^{1}$, $\int_{\phi-1(y)}\left|v_{t_{k}}-v\right| d H^{n-1} \rightarrow 0$ as $t_{k} \rightarrow 0$, and this will be sufficient to establish (5.8). Define

Choose the sequence $t_{k}$ so that $\int_{\Omega}\left|v_{t_{k}}-v\right||\nabla \phi|<2^{-2 k}$ and let $f_{k}=\left|v_{t_{k}}-v\right|$.

$$
A_{k}=\left\{y: \int_{\phi^{-1}(y)}\left|v_{t_{k}}-v\right| d H^{n-1}>2^{-k}\right\}, \quad E=\bigcap_{j=1}^{\infty} \bigcup_{k=j}^{\infty} A_{k} .
$$

Then, from (5.9)

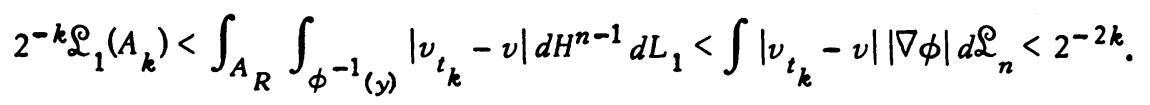


Hence, for any $j, L_{1}(E) \leq \bigcup_{k=j}^{\infty} A_{k} \leq 2^{-j}$, or $L_{1}(E)=0$. But clearly, for $y \notin E$, $\int_{\phi-1(y)}\left|v_{t_{k}}-v\right| d H^{n-1} \rightarrow 0$ as $t_{k} \rightarrow 0$. Thus, we have the following:

Theorem 5.3. Suppose $0>\operatorname{div}^{*}(x) \geq \operatorname{div}_{*} v(x)>-\infty$ for every $x \in \Omega$ and assume that $\operatorname{div}^{*} v$ and $\operatorname{div}_{*} v$ are integrable over $\Omega$. Then $\operatorname{div} v=\operatorname{div}^{*} v=$

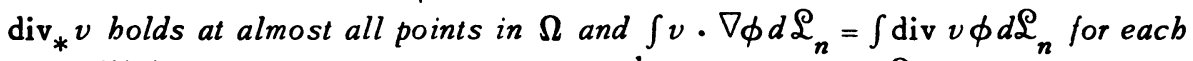
$\phi \in C_{0}^{\infty}(\Omega)$. Moreover, $\left.\int_{\phi-1}(y) v \cdot \nu d H^{n-1}=\int_{\{\phi>y}\right\} \operatorname{div} v d \mathcal{L}_{n}$ for $L_{1}$ almost all $y \in R^{1}$.

It can be shown that the second equality still holds if $\phi$ is assumed only to be Lipschitz or even a continuous Sobolev function. For this purpose the measure theoretic exterior normal must be employed; see below.

By employing standard techniques (see [L], [AP]) we obtain the following.

Corollary 5.4. Let $K \subset \Omega$ be compact and suppose $v \in L_{p}(\Omega-K), p>1$. If the conditions of Theorem 5.3 are satisfied on $\Omega-K$ and if $B_{1, q}(K)=0$, $1 / p+1 / q=1$, then $\int v \cdot \nabla \phi d L_{n}=\int \operatorname{div} v \phi d L_{n}$ for each $\phi \in C_{0}^{\infty}(\Omega)$.

We will now consider the consequences of Theorem 5.3 in the event $v$ is assumed to be defined and continuous everywhere on the closure of $\Omega$. First, consider a set $E$ whose closure is contained in $\Omega$ and for which the GaussGreen theorem is valid. For this purpose we may take $E$ to be a set of finite perimeter, see $[F, \S 4.5]$. That is, the partial derivatives of the characteristic function of $E$ are measures in the sense of distributions. Moreover, there is a number $M$ such that

$$
\lim _{t \rightarrow 0^{+}} \int\left|\nabla\left(\phi_{t} * \chi_{E}\right)\right| d \AA_{n} \leq M
$$

where $\chi_{E}$ is the characteristic function of $E$ and $\phi_{t}$ is the mollifier, as in $\$ 4$. Let $\theta_{t}=\phi_{t} * \chi_{E}$. In view of (5.10), there is a sequence $\left\{t_{k}\right\}$ and a vector valued measure $\sigma$ such that

$$
\int w \cdot \nabla \theta_{t_{k}} d \AA_{n} \rightarrow \int w \cdot d \sigma \text { as } k \rightarrow \infty
$$

for every continuous vector field $w: \Omega \rightarrow R^{n}$. Note, for every smooth vector field $w$, that

$$
\int w \cdot \nabla \theta_{t_{k}} d £_{n}=\int \operatorname{div} w \theta_{t_{k}} d £_{n} \rightarrow \int_{E} \operatorname{div} w ;
$$

and

$$
\int_{E} \operatorname{div} w=\int_{\partial * E} w \cdot \nu d H^{n-1}
$$

where $\partial^{*} E$ is the set of points $x$ in the boundary of $E$ where the exterior normal $\nu(x)$, which is defined in the measure theoretic sense, exists, [F, Theorem 4.5.6]. 
Thus, (5.11) and (5.12) imply that

$$
\int_{E} \operatorname{div} v d \complement_{n}=\int v \cdot d \sigma,
$$

whereas (5.13) and (5.14) show $\int w \cdot d \sigma=\int_{\partial}{ }^{*} E \cdot \nu d H^{n-1}$ for every smooth $w$, and therefore for every continuous $w$. Thus, it follows from (5.14) that

$$
\int_{E} \operatorname{div} v d \AA_{n}=\int_{\partial * E} v \cdot \nu d H^{n-1}
$$

whenever $E$ is a set of finite perimeter whose closure is contained in $\Omega$.

We are now in a position to prove the following theorem which is an extension of a result due to Shapiro [SH].

Theorem 5.5. Let $\Omega \subset R^{n}$ be a bourded open set with $H^{n-1}(\partial \Omega)<\infty$ and let $K \subset \Omega$ be compact. Suppose $v$ is a vector field which is continuous on closure $\Omega$ and is in $L_{p}(\Omega-K), p>1$. Suppose $\infty>\operatorname{div}^{*} v(x) \geq \operatorname{div}_{*} v(x)>-\infty$ for every $x \in \Omega-K$ and that $\operatorname{div}^{*} v$ and $\operatorname{div}_{*} v$ are integrable over $\Omega$. If $B_{1, q}(K)=0$, $1 / p+1 / q=1$, then $\int_{\Omega} \operatorname{div} v d \mathfrak{L}_{n}=\int_{\partial * \Omega} \nu \cdot \nu d H^{n-1}$.

Proof. In view of the hypothesis that $\partial \Omega$ is a compact set with $H^{n-1}(\partial \Omega)<\infty$, it follows there is a number $M>0$ such that, for every $\epsilon>0$, there is a finite number of open $n$-balls, $B_{1}, B_{2}, \ldots, B_{k}(k=k(\epsilon))$ with $\operatorname{diam} B_{i}<\epsilon, \partial \Omega \subset \bigcup_{i=0}^{k} B_{i}$, and $\sum_{i=1}^{k} H^{n-1}\left(\partial B_{i}\right) \leq M$. Let $\Omega_{\epsilon}=\Omega-\bigcup_{i=1}^{k} B_{i}$ and observe that $H^{n-1}\left(\partial \Omega_{\epsilon}\right) \leq M$. Thus, $\Omega_{\epsilon}$ has finite perimeter and Theorem 5.5 implies $\int_{\Omega_{\epsilon}} \operatorname{div} v d \mathfrak{Q}_{n}=$ $\int_{\partial^{*} \Omega_{\epsilon}} v \cdot \nu d H^{n-1}$. Since $H^{n-1}\left(\partial^{*} \Omega_{\epsilon}\right) \leq H^{n-1}\left(\partial \Omega_{\epsilon}\right) \leq M$ for all $\epsilon>0$, there is a vector valued measure $\sigma$ such that, for some sequence $\epsilon_{i} \rightarrow 0$,

$$
\int_{\partial * \Omega_{\epsilon}} w \cdot \nu d H^{n-1} \rightarrow \int w \cdot d \sigma
$$

whenever $w$ is a continuous vector field with compact support. As in the proof of Theorem 5.5 we can show that $\int w \cdot d \sigma=\int_{\partial *} \Omega w \cdot \nu d H^{n-1}$ for every continuous $w$ and therefore $\int_{\Omega} \operatorname{div} v d \mathscr{\complement}_{n}=\int_{\partial *} \Omega v \cdot \nu d H^{n-1}$.

\section{REFERENCES}

[AP] D. Adams and J. Polking, The equivalence of two definitions of capacity, Proc. Amer. Math. Soc. 37 (1973), 529-535.

[AS] N. Aronszajn and K. Smith, Functional spaces and functional completion, Ann. Inst. Fourier (Grenoble) 6 (1955/56), 125-185. MR 18, 319.

[B] S. Bochner, Green-Goursat theorem, Math. Z. 63 (1955), 230-242. MR 17, 250.

[C1] A. P. Calderón, Lebesgue spaces of differentiable functions and distributions, Proc. Sympos. Pure Math., vol. 4, Amer. Math. Soc. Providence R. I., 1961, pp. 33-49. MR $26 \# 603$.

[C2] A. P. Calderón, Uniqueness of distributions, Rev. Un. Mat. Argentina 25 (1971).

[CZ] A. P. Caldeón and A. Zygmund, Local properties of solutions of elliptic partial differential equations, Studia Math. 20 (1961), 171-225. MR 25 \#10. 
[F] H. Federer, Geometric measure theory, Die Grundlehren der math. Wissenschaften, Band 153, Springer-Verlag, New York, 1969. MR 41 \#1976.

[FZ] H. Federer and W. P. Ziemer, The Lebesgue set of a function whose distribution derivatives are p-th power summable, Indiana Univ. Math. J. 22 (1972), 139-158.

[FU] B. Fuglede, On a theorem of F. Riesz, Math. Scand. 3 (1955), 283-302. MR $18,198$.

[G] C. Goffman, On functions with summable derivatives, Amer. Math. Monthly 78 (1971), 874-875. MR 44 \#4156.

[L] W. Littman, Polar sets and removable singularities of partial differential equations, Ark. Mat. 7 (1967), 1-9.

[M1] N. G. Meyers, A theory of capacities for potentials of functions in Lebesgue classes, Math. Scand. 26 (1970), 255-292. MR 43 \#3474.

[M2] —_, Taylor expansion of Bessel potentials, Indiana Univ. Math. J. (to appear).

[MO] C. B. Morrey, Multiple integrals in the calculus of variations, Die Grundlehren der. math. Wissenschaften, Band 130, Springer-Verlag, New York, 1966. MR 34 \#2380.

[N] C. J. Neugebauer, Smoothness and differentiability in $L_{p}$, Studia Math. 25 (1964/65), 81-91. MR 31 \#5942.

[SA] S. Saks, Theory of the integral, 2nd rev. ed., Monografie Mat., vol. 7, PWN, Warsaw, 1937.

[SE] J. Serrin (to appear)

[SH] V. Shapiro, The divergence theorem for discontinuous vector fields, Ann. of Math. (2) 68 (1958), 604-624. MR 20 \#7153.

[S] E. Stein, Singular integrals and differentiability properties of functions, Princeton Math. Series, no. 30, Princeton Univ. Press, Princeton, N. J., 1970. MR 44 \#7280.

DEPARTMENT OF MATHEMATICS, INDIANA UNIVERSITY, BLOOMINGTON, INDIANA 47401 